\title{
Bilateral isolated olecranon fractures after trauma: Report of two cases
}

\author{
Atilla Çıtlak $^{a^{*}}$, Nizamettin Güzel ${ }^{\mathrm{b}}$, Servet Kerimoğlu ${ }^{\mathrm{c}}$, Osman Aynacı ${ }^{\mathrm{c}}$ \\ ${ }^{a}$ Department of Orthopaedics and Traumatology, Faculty of Medicine, Giresun University, Giresun, Turkey \\ ${ }^{b}$ Department of Orthopaedics and Traumatology, Kelkit State Hospital, Gümüşhane, Turkey \\ ${ }^{c}$ Department of Orthopaedics and Traumatology, Faculty of Medicine, Karadeniz Technical University, Trabzon, Turkey
}

\section{ARTICLE INFO}

\section{Article History}

Received $\quad 01 / 10 / 2013$

Accepted $\quad 05 / 10 / 2013$

\section{* Correspondence to:}

Atilla Çitlak

Department of Orthopaedic Surgery, Faculty of Medicine,

Giresun University,

Giresun, Turkey

e-mail: atillacitlak@yahoo.com

\section{Keywords:}

Bone fractures

Elbow joint

Olecranon

Trauma

\section{ABSTRACT}

Olecranon fractures represent approximately $10 \%$ of all fractures around the elbow in adults. Olecranon fractures occur by a fall or blunt trauma on the posterior tip of elbow, or by the forces generated by triceps muscle during a fall. Olecranon fractures were seen as unilateral fractures, however bilateral olecranon fractures were seen as pathological fractures. Anatomic restoration of the articular surface, repair of the extensor mechanism and full range of motion with stability are the main goals of treatment in olecranon fractures. Operative management should be performed when there is any articular incongruity or extensor mechanism deficit. Tension band fixation, intramedullary fixation, plating and proximal fragment excision are the main operative techniques which were used for the olecranon fractures. In this study, we reported two cases of bilateral traumatic olecranon fractures in patients without any concomitant systemic disease. Tension band wiring technique was used for the treatment of bilateral olecranon fractures. We preferred transcortical tension band wiring because it serves adequate stabilization and fragment compression with minimal instrumentation. When we reviewed the literature, we could not find isolated bilateral olecranon fractures in a patient without any concomitant disease. Treatment and rehabilitation of bilateral fractures served successful results with tension band wiring as unilateral fractures.

J. Exp.Clin.Med., 2014; 31:107-110

(C) $2014 \mathrm{OMU}$

\section{Introduction}

Olecranon fractures represented approximately $10 \%$ of all fractures around the elbow in adults (Veillette and Steinmann, 2008; Wiegand et al., 2012). Most commonly olecranon fractures were seen as isolated injuries (Wiegand et al., 2012). Olecranon fractures occur by a fall or blunt trauma on the posterior tip of elbow (direct trauma), or by the forces generated by triceps muscle during a fall (indirect trauma) (Veillette and Steinmann, 2008). There are several classification systems for olecranon fractures (Veillette and Steinmann, 2008; Newman et al., 2009; Wiegand et al., 2012). The Mayo classification of olecranon fractures is the mostly preferred one (Veillette and Steinmann, 2008; Wiegand et al., 2012). According to this classification system Type I fractures are undisplaced, Type II fractures are stable fractures with displacement, Type III fractures are unstable, displaced fracture-dislocations (Veillette and Steinmann, 2008; Newman et al., 2009; Wiegand et al., 2012). Olecranon fractures were seen as unilateral fractures, however bilateral olecranon fractures were seen as pathological fractures (Kirmani et al., 2008; O’Daly et al., 2008; Wiegand et al., 2012).

When we reviewed the literature, we could not find isolated bilateral olecranon fractures in the same patient without any concomitant disease. We report two cases of bilateral traumatic olecranon fractures in patients without any concomitant systemic disease.

\section{Case Reports}

Case 1

An eighteen-year-old man admitted to emergency department with pain and edema in his elbow joints after a fall while going down the stairs. Physical examination revealed deformity and swelling of both elbows. Neurovascular examination was normal and the skin of the elbows was intact. Direct radiographs revealed olecranon fractures in both elbows, 
according to Mayo classification type II a in right elbow, type II b in left elbow (Fig. 1A). The patient was prepared for surgery. Under general anesthesia in supine position right olecranon fracture was opened by posterior skin incision. The fracture was explored and hematoma was washed using saline. After reduction two kirshner wires were used for stabilization of the fracture. Kirshner wires were inserted transcortically without penetrating the articular cartilage. The wires were bent and cut. Posterior cortex of ulna was drilled, and then a wire was passed from this hole. The fracture line then is compressed with this figure-eight of wire. The same tension band wiring technique was used for left olecranon (Fig. 1B). Both elbows were splinted with the elbow flexed 90 degrees in the operating room. After seven days splints were removed and passive motion and gentle active assisted motion were started. The wires were removed after four months. After 18 months, the patient had full range of motion; there was no loss of any motion in both elbows (Fig. 1C).

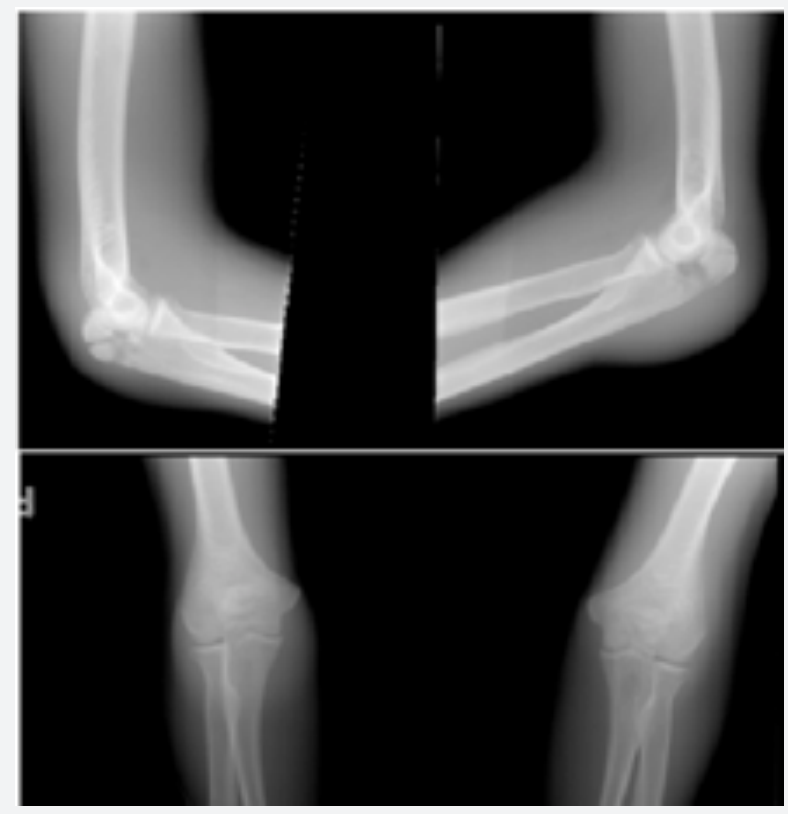

Fig. 1A. Preoperative direct X-rays of first patient.
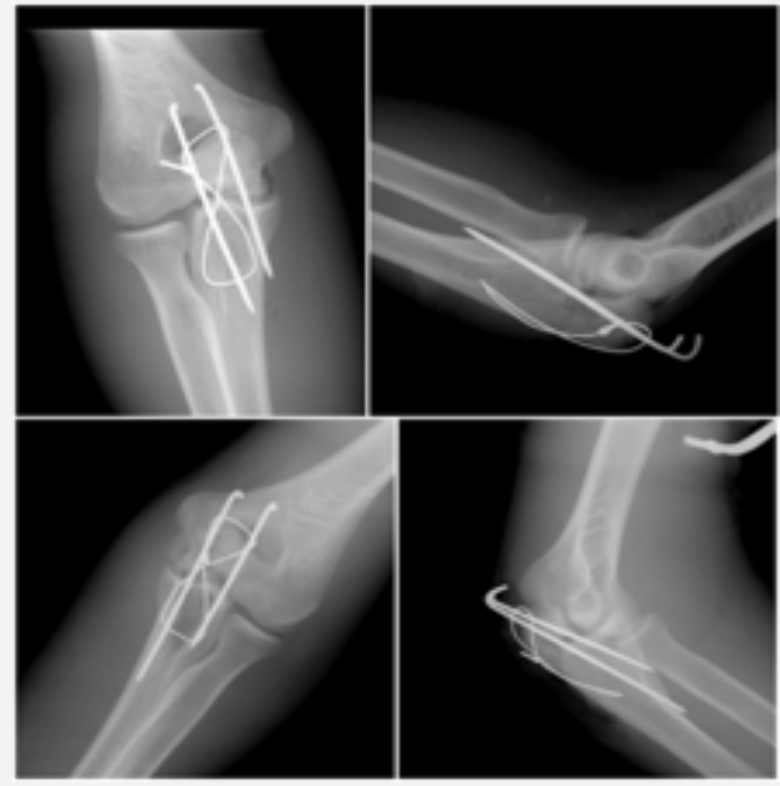

Fig. 1B. Postoperative direct X-rays of first patient.

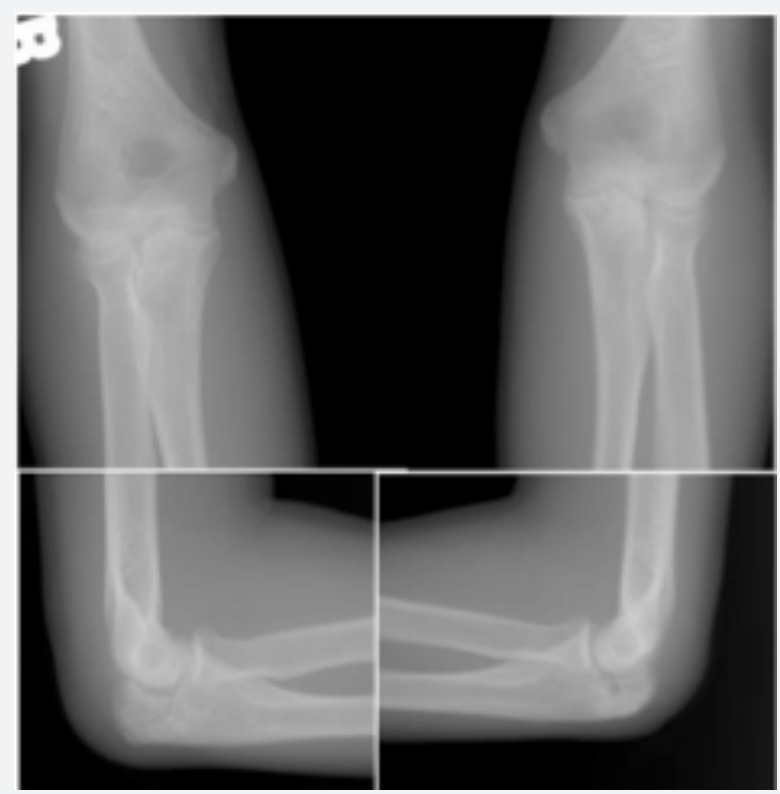

Fig. 1C. After 18 months direct X-rays of first patient.

Case 2

A seventeen-year-old woman admitted to emergency department after a fall from first floor. The patient was conscious and there was pain in both elbows. On physical examination crepitation and edema were found in both elbow joints. The pulses were present, and skin was intact in all joints. Neurological examination was normal. Direct radiography revealed bilateral olecranon fractures (Fig. 2A, B). Open reduction internal fixation was planned for these fractures. Tension band wiring technique was used for bilateral olecranon fractures as the first patient (Fig. 2C, D). After seven days motion was started. The wires were removed after four months. After one year, the patient was symptom free and had full range of motion in both elbows (Fig. 2E, F).

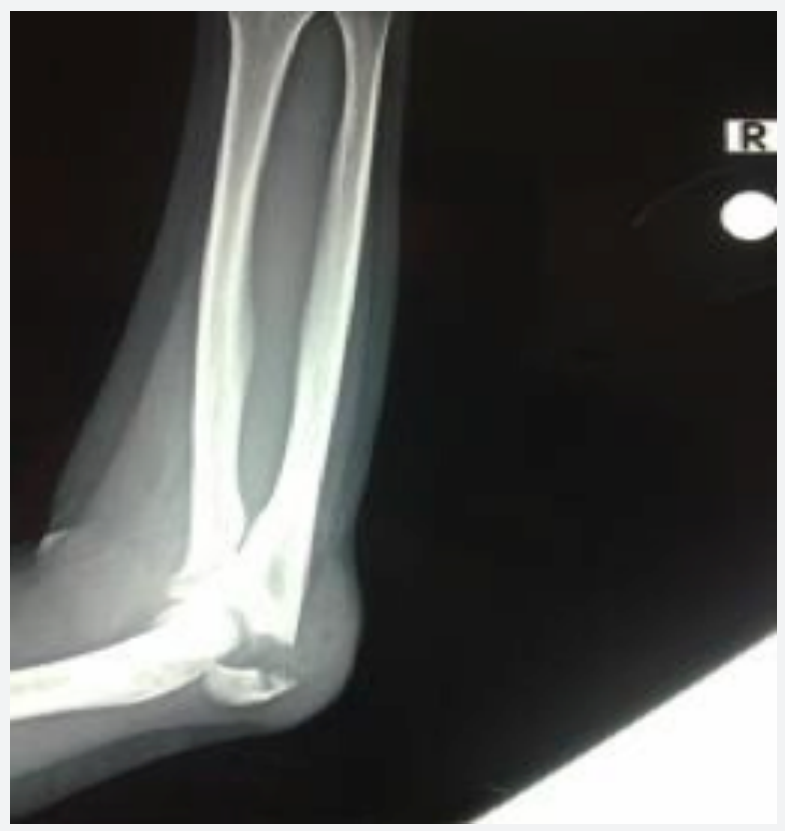

Fig. 2A. Preoperative lateral X-ray of right elbow of second patient. 


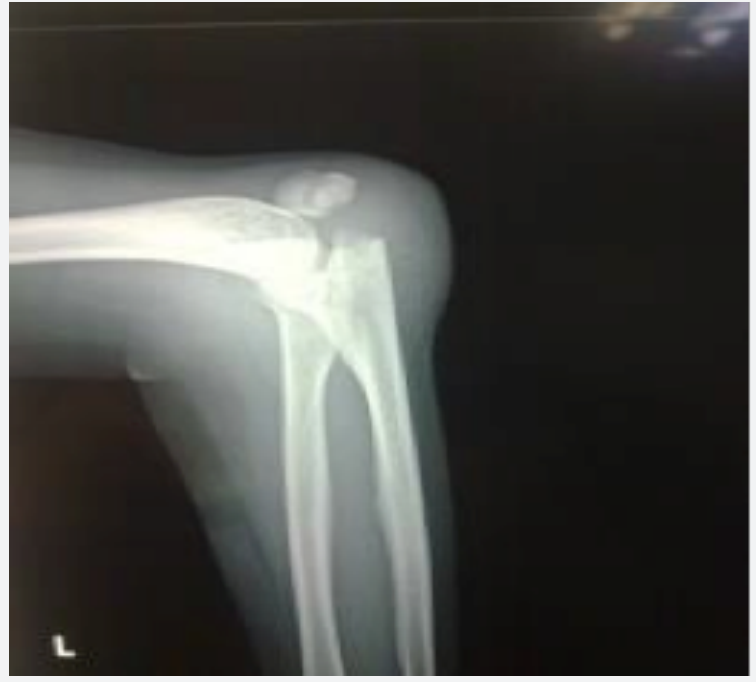

Fig. 2B. Preoperative lateral X-ray of left elbow of second patient.

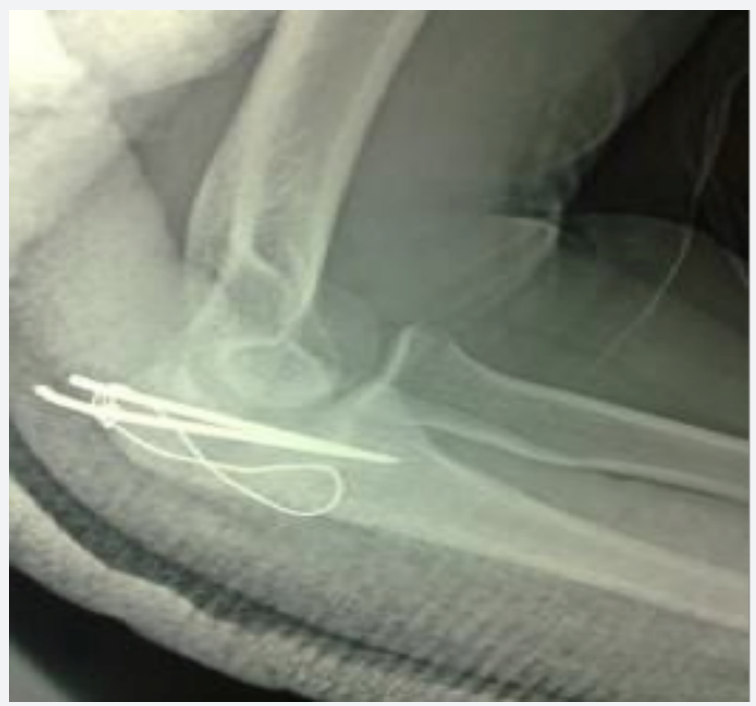

Fig. 2C. Postoperative lateral X-ray of right elbow of second patient.

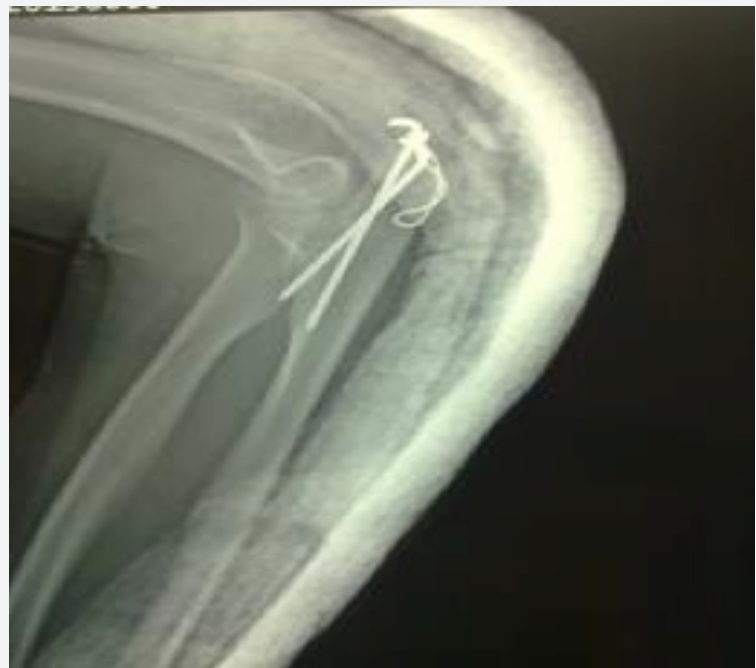

Fig. 2D. Postoperative lateral X-ray of left elbow of second patient.

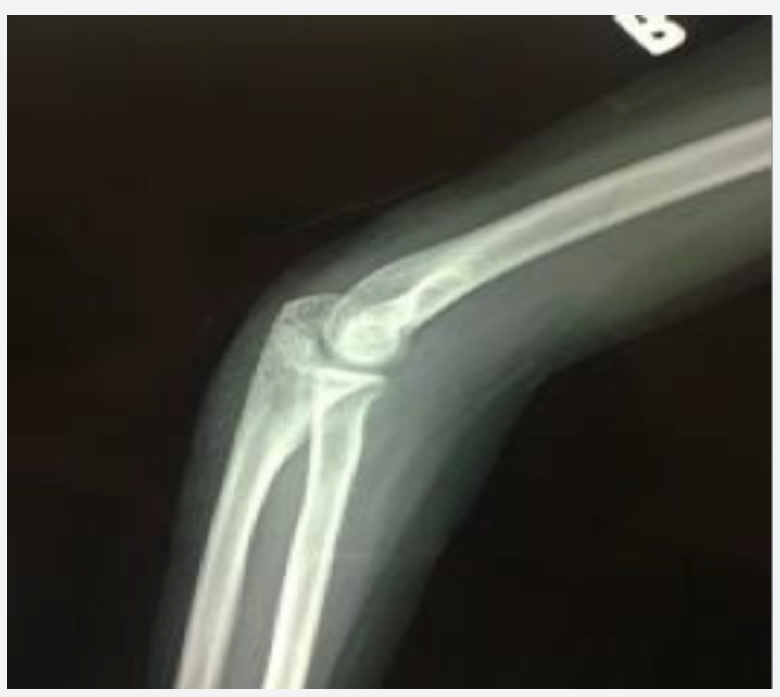

Fig. 2E. After one year lateral X-ray of right elbow of second patient.

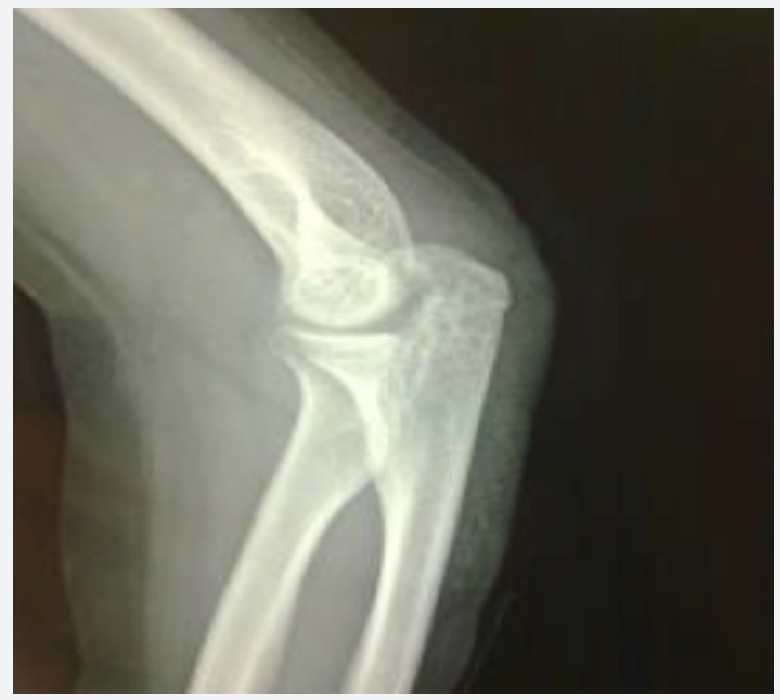

Fig. 2F. After one year lateral X-ray of left elbow of second patient.

\section{Discussion}

Olecranon fractures represent a common fracture treated by orthopaedic surgeons (Donegan and Bell, 2010). Direct trauma or indirect trauma can produce olecranon fracture (Veillette and Steinmann, 2008). Generally comminuted fractures are as a result of direct trauma, and transverse fractures are as a result of indirect trauma (Newman et al., 2009).

Anatomic restoration of the articular surface, repair of the extensor mechanism and full range of motion with stability are the main goals of treatment in olecranon fractures (Wiegand et al., 2012). All olecranon fractures are intra articular (Newman et al., 2009). There are various treatment options. Non operative management generally preferred for undisplaced fractures and in elderly patients with significant displacement (Newman et al., 2009). Operative management should be performed when there is any articular incongruity or extensor mechanism deficit (Wiegand et al., 2012).

Tension band fixation, intramedullary fixation, plating and 
proximal fragment excision are the main operative techniques which were used for the olecranon fractures (Newman et al., 2009). Tension band wiring technique is a widely accepted technique which yields good and excellent results (Linden et al., 2012; Villanueva et al., 2006). Transcortical placement of Kirshner wires serve more stable fracture fixation (Linden et al., 2012). We preferred transcortical tension band wiring because it serves adequate stabilization and fragment compression with minimal instrumentation. Also hardware removal is easy when compared to other methods. Prominent hardware related complaints have been reported in all fixation techniques (Veillette and Steinmann, 2008). Tension band wiring technique offers excellent results despite the associated complications (Donegan and Bell, 2010). Pain on the Kirshner wire prominence is the most common complication of tension band wiring (Donegan and Bell, 2010). After the fracture union the hardware was removed in both cases. Most patients heal well with approximately $1 \%$ nonounion, and some loss of terminal extension (Wiegand et al., 2012). Both cases healed without any complication.

Olecranon fractures most commonly occur as isolated injuries, but it is important to evaluate other injuries especially in the ipsilateral extremity (Wiegand et al., 2012). Additional lesions in the ipsilateral extremity had a negative effect on range of motion, and serve more complications (Rommens et al., 2004). Bilateral fractures might be caused by high energy traumas and free fall injuries, but in those patients other parts of the body should be fractured. We determined only bilateral isolated olecranon fractures with fall.

Bilateral olecranon fractures were seen as pathologic fracture in a rheumatoid patient (Kirmani et al., 2008). Due to age and osteopenic nature of bones, the patient had spontaneous bilateral olecranon fracture (Kirmani et al., 2008). Another case with bilateral pathologic fractures of the olecranon, occurring within a month period of each other, presented with a painful swollen elbow (O'Daly et al., 2008). Bilateral olecranon fractures were the first presentation of sarcoidosis in that patient (O'Daly et al., 2008). Bilateral olecranon fractures could be seen as pathological fractures in patients with systemic diseases, but we reported bilateral olecranon fractures after trauma in patients without any disease. Treatment modalities which were used in unilateral fractures served successful results in bilateral fractures. Bilateral fracture did not change the treatment result.

When we reviewed the literature we could not find isolated bilateral olecranon fractures in the same patient without any concomitant disease. Treatment and rehabilitation of bilateral fractures served successful results with tension band wiring as unilateral fractures.

\section{REFERENCES}

Donegan, R.P., Bell, J.E., 2010. Olecranon fractures. Oper. Tech. Orthop. 20, 17-23.

Kirmani, S., Draviaraj, K., Madegowda, B., Shahane, S., 2008. Spontaneous bilateral olecranon fractures in a rheumatoid patient. Ann. R. Coll. Surg. Engl. 90, 1-3. doi: 10.1308/147870808X257256.

Linden, S.C., Kampen, A., Jaarsma, R.L., 2012. K-wire position in tension-band wiring technique affects stability of wires and long-term outcome in surgical treatment of olecranon fractures. J. Shoulder. Elbow. Surg. 21, 405-411. doi: 10.1016/j.jse.2011.07.022.

Newman, S.D., Mauffrey, C., Krikler, S., 2009. Olecranon fractures. Injury. 40, 575-581. doi: 10.1016/j.injury.2008.

O’Daly, B.J., Harty, J.A., O’Malley, N., Killeen, R., McDonnell, T.J., Quinlan, W.R., 2008. Bilateral olecranon fracture as first presentation of sarcoidosis: Case report and review of the literature. J. Shoulder. Elbow. Surg. 17, 1-5. doi: 10.1016/j.jse.2007.06.016.

Rommens, P.M., Küchle, R., Schneider, R.U., Reuter, M., 2004. Olecranon fractures in adults: Factors influencing outcome. Injury. 35, 11491157.

Veillette, C.J., Steinmann, S.P., 2008. Olecranon fractures. Orthop. Clin. N. Am. 39, 229-236. doi: 10.1016/j.ocl.2008.01.002.

Villanueva, P., Osorio, F., Commessatti, M., Sanchez-Sotelo, J., 2006. Tension-band wiring for olecranon fractures: Analysis of risk factors for failure. J. Shoulder. Elbow. Surg. 15, 351-356.

Wiegand, L., Bernstein, J., Ahn, J., 2012. Fractures in brief: Olecranon fractures. Clin. Orthop. Relat. Res. 470, 3637-3641. doi: 10.1007/s11999012-2393-5. 